Review

\title{
The Involving Roles of Intrahepatic and Extrahepatic Stem/Progenitor Cells (SPCs) to Liver Regeneration
}

\author{
Wei-hui Liu ${ }^{1 \# \bowtie}$, Li-na Ren#, Tao Wang"1\#, Nalu Navarro-Alvarez², Li-jun Tang1 \\ 1. General Surgery Center, Chengdu Military General Hospital; Chengdu, Sichuan Province, 610083 \\ 2. Department of Surgery, Massachusetts General Hospital, Harvard Medical School, Boston, Massachusetts, USA. \\ \# These authors equally contributed to this work.
}

$\square$ Corresponding author: Professor Wei-hui Liu. Addresses: General Surgery Center of PLA; Chengdu Military General Hospital, Chengdu, Sichuan Province, 610083; China. Telephone: Fax: 0086-028-86570265; E-mail: audiliu12@163.com (Wei-hui Liu)

(0) Ivyspring International Publisher. Reproduction is permitted for personal, noncommercial use, provided that the article is in whole, unmodified, and properly cited. See http://ivyspring.com/terms for terms and conditions.

Received: 2016.04.01; Accepted: 2016.05.09; Published: 2016.06.18

\begin{abstract}
Liver regeneration is usually attributed to mature hepatocytes, which possess a remarkable potential to proliferate under mild to moderate injury. However, when the liver is severely damaged or hepatocyte proliferation is greatly inhibited, liver stem/progenitor cells (LSPCs) will contribute to the liver regeneration process. LSPCs in the developing liver have been extensively characterized, however, their contributing role to liver regeneration has not been completely understood. In addition to the restoration of the liver parenchymal tissue by hepatocytes or/and LSPCs, or in some cases bone marrow (BM) derived cells, such as hematopoietic stem cells (HSCs) and mesenchymal stem cells (MSCs), the wound healing after injury in terms of angiopoiesis by liver sinusoidal endothelial cells (LSECs) or/and sinusoidal endothelial progenitor cells (SEPCs) is another important aspect taking place during regeneration. To conclude, liver regeneration can be mainly divided into three distinct restoring levels according to the cause and severity of injury: hepatocyte dominant regeneration, LSPCs mediated regeneration, extrahepatic stem cells participative regeneration. In this review, we focus on the recent findings of liver regeneration, especially on those related to stem/progenitor cells (SPCs)-mediated regeneration and their potential clinical applications and challenges.
\end{abstract}

Key words: liver stem/progenitor cells (LSPCs); sinusoidal endothelial progenitor cells (SEPCs); mesenchymal stem cells (MSCs); hematopoietic stem cells (HSCs); liver regeneration

\section{Introduction}

The liver has an enormous capacity to regenerate through different cellular responses depending on the nature and severity of the injury [1]. The whole process of liver repair can be integrated into two aspects including regeneration and wound healing [2]. In other words, in the context of liver injury, successful repair consists of both efficient parenchymal restoration and appropriate angiogenesis which are sufficient to promote liver regeneration in most cases [3]. Both aspects are modulated by a dynamic interplay between parenchymal and non-parenchymal cells, including hepatocytes [4], hepatic stellate cells $[5,6]$, inflammatory cells [7], cholangiocytes [8], and liver sinusoidal endothelial cells (LSECs) $[9,10]$. Therefore, defining the multi-cellular crosstalk that balances regeneration and dysfunctional healing holds promise to design new treatments for liver diseases.

Liver regeneration is a relatively complex process and requires the coordinated contribution of different cell types [4]. Generally speaking, due to different liver injuries, liver regeneration can be mainly divided into three distinct restoring levels [11]. Under normal conditions, or after moderate loss of parenchyma by surgical resection, liver mass is maintained by division of hepatocytes $[12,13]$. In addition to mature intrahepatic cells, resident and migrating stem/progenitor cells (SPCs) also actively 
participate in liver regeneration. For example, activation of liver stem/progenitor cells (LSPCs) is widely identified in both acute and chronic injuries $[14,15]$. When hepatocyte proliferation is inhibited or overwhelmed, liver injury leads to the activation of LSPCs to replace the injured hepatic parenchyma [14, 15]. Hepatic oval cells (HOCs) and small hepatocyte-like progenitor cells (SHPCs) are two of the LSPC populations that have the capacity to differentiate into both hepatocyte and cholangiocytes [16]. HOCs or SHPCs rapidly proliferateand differentiate into hepatocytes and cholangiocytes to compensate for the cellular loss and maintain liver homeostasis. In addition to intrahepatic SPCs, bone marrow (BM) derived SPCs can also contribute to liver regeneration by the fusion of mesenchymal stem cells (MSCs) with damaged hepatocytes, or differentiation into hepatocyte-like cells [17]. In this review, we aimed to briefly introduce the proved mechanism involving in liver regeneration, especially the contributing responses of SPCs.

\section{The contributing roles of LSPCs participating in liver regeneration}

\section{The proof for LSPCs participating in liver regeneration}

LSPCs are very different from tissue-resident stem cells in other epithelial tissue such as intestine and skin [18]. In the latter tissues, SPCs are required throughout life to replenish cells lost daily, and without continued replication of the tissue-resident stem cells, the epithelia of intestine and skin fail rapidly. In contrast, LSPCs are "facultative," meaning [19] (1) they are not needed to fill up liver tissue under normal, healthy conditions and (2) many markers of LSPCs are only expressed in the liver after injury when SPCs are activated. In severely damaged livers when there is a massive loss and/or an inhibited proliferative capacity of hepatocytes, activation of resident LSPCs occurs to contribute to regeneration [19]. When activated, LSPCs migrate into the hepatic lobules and differentiate into hepatocytes or cholangiocytes to support or take over the role of regeneration $[14,15]$.
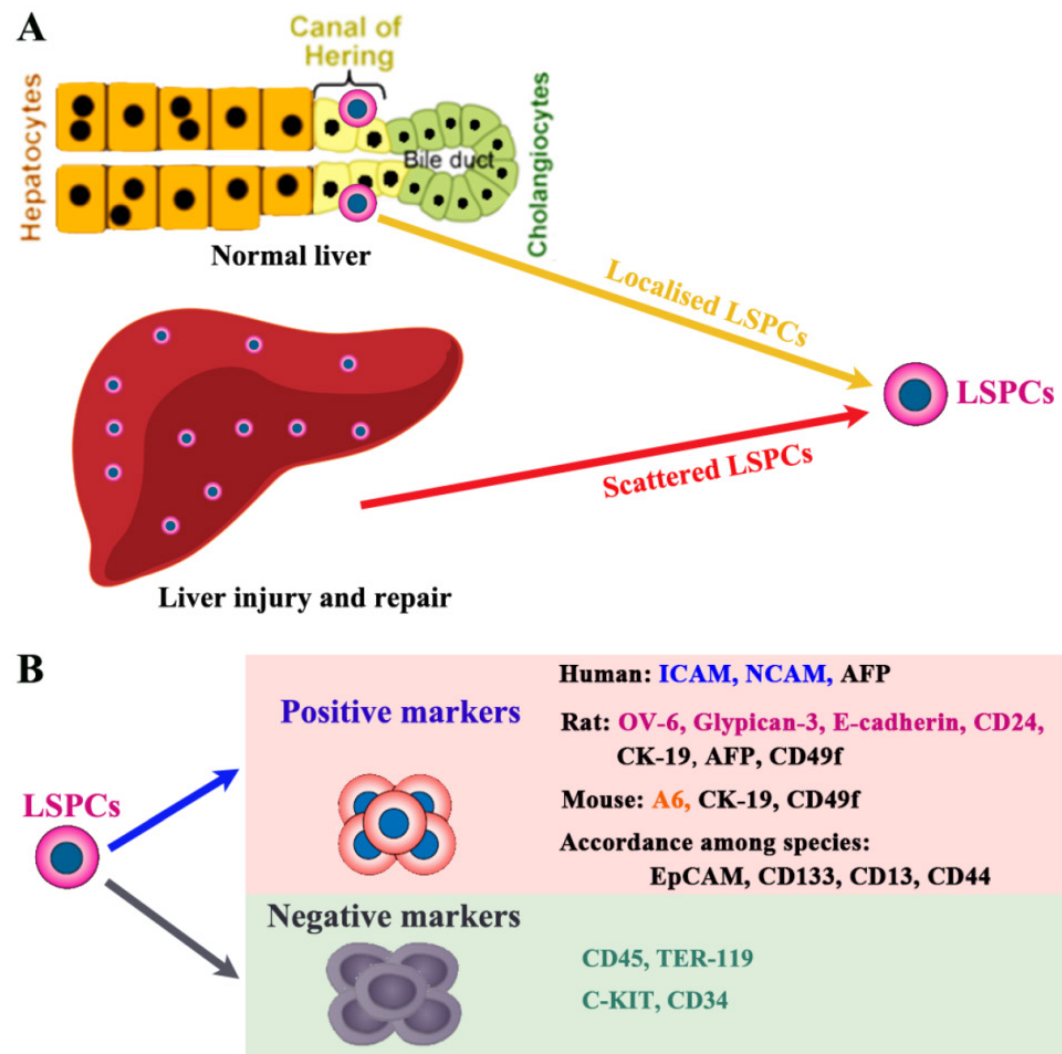

Figure 1. The localization and cell surface markers for liver stem/progenitor cells (LSPCs). (A) So far, the origin of LSPCs is still not clear. Nevertheless, there is a widely accepted theory that LSPCs are derived from the canal of Hering, where the putative "resident" stem cells aggregate as the exact cell of origin. In addition, upon certain types of injury condition, LSPCs may expand and scatter in the liver parenchyma. (B) The representative intracellular and surface markers among species are shown. For human beings, rats and mice, LSPCs share some markers, however, there are differently expressed cell markers among different species. The most compelling markers potentially useful for isolation of LSPCs in different species, are shown.

\section{The localization and isolation of LSPCs}

LSPCs reside in the terminal branches of the intrahepatic biliary tree (Canal of Hering) in normal liver (Figure 1A) [20]. Recently, using Wnt-responsive gene Axin2 in mice, Wang et al. identify a population of LSPC-like cells adjacent to the central vein in the liver lobule [21]. During liver injury and repair process, some LSPCs also appear scattered in the parenchyma. However, the lack of highly specific markers has hampered efforts to better understand the origin and physiology of LSPCs [22]. Here we only briefly describe the potential markers used to detect or isolate LSPCs (Figure 1B), which is systematically described in our previous review [23]. For sorting LSPCs, the following markers have been demonstrated effective, c-Kit-/low [24], c-Kit-c-Met+CD49f+/ low [25], CD13+ [26], CD13+c-Kit-CD49f-/lowCD133+ [27], or CD24+CK19+ [28] in combination with the non-hematopoietic marker CD45-TER119-. This CD24+ CK19+CD45-Ter119- cell population represented $0.04 \%$ of liver cells and 
expressed several markers of LSPCs, such as CD133, Dlk, and Sca-1 high, but c-Kit, Thymus cell antigen-1 (Thy-1), and CD34 low. In liver damage condition, single cell marker is also widely used to screen LSPCs. For example, it is demonstrated that Lgr5 ((leucine-rich-repeat-containing G-protein-coupled receptor 5)+ small cells near bile ducts actively take part in the liver regeneration after damage by generating significant numbers of hepatocytes and biliary duct cells [29].

\section{The experimental models for mobilization of LSPCs}

Partial hepatectomy (PHx) is considered as a classical model for studying liver regeneration in mammals [14]. In those damaged livers where hepatocyte proliferation is compromised, LSPCs are activated and differentiate into hepatocytes and cholangiocytes, leading to functional recovery of the organ. In humans, a minimum of $50 \%$ hepatocyte loss is required for significant activation of the LSPC compartment [19], and there is an inverse correlation between the number of LSPCs and the number of proliferating hepatocytes with Ki67 expression [30]. This suggests that a combination of hepatocyte loss and impaired hepatocyte proliferation is required to activate LSPC. For stimulating LSPCs to participate in liver regeneration in rodents, a number of models have been applied (Figure 2). The most popular model to induce LSPCs is the combination of PHx with chemical inhibition of hepatocyte proliferation using 2-acetylaminofluorene (2-AAF) or retrorsine in rats, where hepatocyte proliferation is blocked by 2-AAF before and after PHx while inducing a robust LSPCs response [31,32]. However, in mice this system is not applicable because it fails to produce convincing activation of LSPCs, instead, several other dietary or toxin models of LSPC activation have been described. In short, the administration of a 3,5-diethoxycarbonyl1,4-dihidro-collidine (DDC)-containing diet [33, 34] or a choline-deficient, ethionine-supplemented (CDE) diet $[34,35]$ is the most extensively used model for activating LSPCs.

\section{The possible mechanisms responsible for LSPCs mediated liver regeneration}

The LSPC response can be divided into four phases: activation, proliferation, migration and differentiation (Figure 3), the final step leading to either hepatocytes or cholangiocytes. The LSPC niche comprises the parenchymal and non-parenchymal resident liver cells, recruited inflammatory cells, extracellular matrix (ECM) as well as the variety of growth-regulating factors [14, 33, 36-38], which together control the LSPC response to liver regeneration.

\section{The affecting cells of LSPC response in liver regeneration}

The LSPC niche is composed of a large variety of cell types (Figure 3A) such as hepatic stellate cells, LSECs, hepatocytes, cholangiocytes, Kupffer cells, pit cells and inflammatory cells [39]. All these cell types, numerous hormones and growth factors could interact and cross-talk with LSPCs influencing their proliferative and differentiative processes (Figure 3B). For instance, macrophages and T-cells have been shown to interact with LSPCs to regulate proliferation, differentiation, and migration of LSPCs [40-42], while hepatic stellate cells or myofibroblasts have also been observed in close proximity of LSPCs both in mouse [43] and in patients with liver diseases [44]. The hepatic stellate cells are major resident non-parenchymal liver cells [45]. During the early

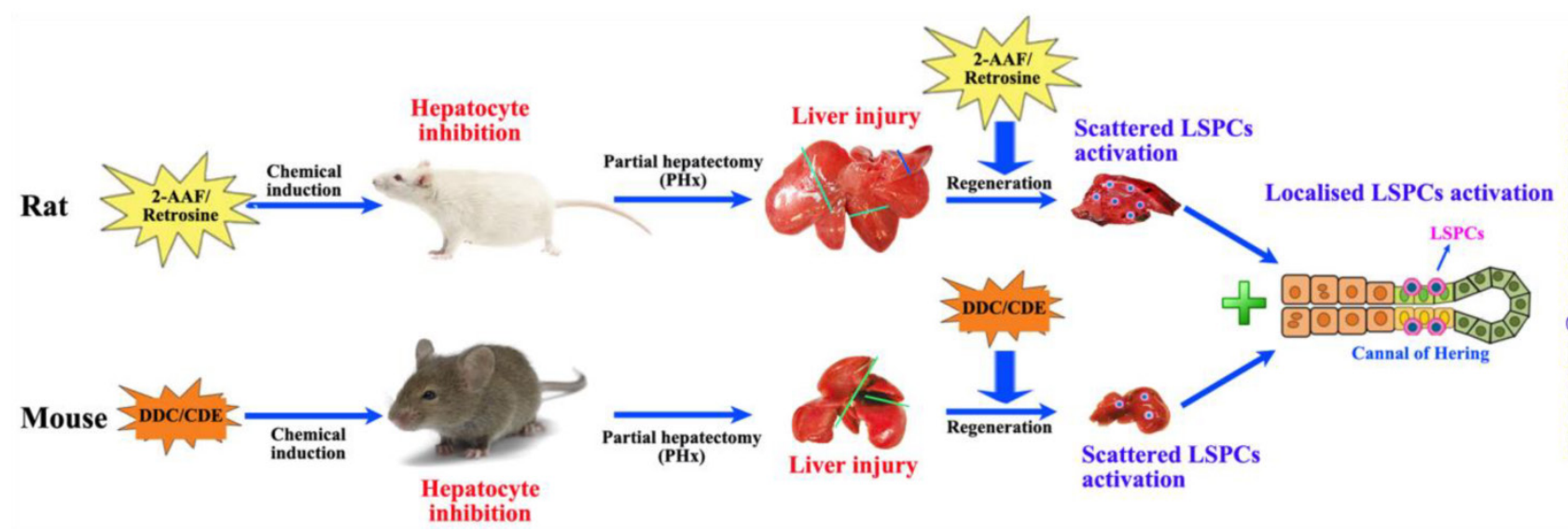

Figure 2. Two modes of LSPCs mediated liver regeneration in rats and mice. Both in rats and in mice, partial hepatectomy (PHx) is applied to cause liver injury for the remaining tissue to enlarge and recover the original mass. This type of regeneration is mainly accomplished by mature hepatocytes in the remaining tissue. When PHx combines with the impaired proliferative capacity of hepatocytes by treatment with 2-acetylaminofluorene (2-AAF)-containing diet in rats and 3,5-diethoxycarbonyl-1,4-dihidro-collidine (DDC)-containing diet or a choline-deficient, ethionine-supplemented (CDE) diet in mice, immature LSPCs are activated and contribute to the regeneration process by differentiating to hepatocytes and cholangiocytes. 
period of liver regeneration (2-6 days after injury), it was found that hepatic stellate cells secreted high levels of hepatocyte growth factor (HGF) and stimulated LSPC proliferation via extracellular signal-regulated kinase and p38 pathway, whereas in the terminal phase of liver regeneration (12 and 15 days group) hepatic stellate cells generated high levels of transforming growth factor (TGF)- $\beta 1$, which in turn suppressed LSPCs' DNA synthesis. These results indicate that hepatic stellate cells have the dual role of promoting and inhibiting LSPCs proliferation during the liver regeneration process [46], act as a positive regulator at the early phase and a negative regulator at the terminal phase of the LSPC-mediated liver regeneration. The shift between these two distinct effects depends on the balance between HGF and TGF- $\beta 1$ secreted by hepatic stellate cells.

In addition, it has been demonstrated that Thy-1+ mesenchymal cells are also part of the LSPCs niche $[47,48]$. In all models of LSPC-mediated liver regeneration they proliferate quickly and expand significantly and disappear from the liver when the LSPC response subsides. Activated Thy-1+ cells do not express LSPC genes but they express other genes (CD105, CD73, CD29) known to be expressed in MSCs, genes considered specific for activated hepatic stellate cells (desmin, collagen I- 2 2, matrix metalloproteinases (MMP)2, MMP14) and myofibroblasts [alpha smooth muscle actin (alpha-SMA), fibulin-2], as well as growth factors and cytokines (HGF, tumor necrosis factor (TNF)-like weak inducer of apoptosis (TWEAK), interleukin (IL)-1 $\beta$, IL-6, IL-15) that can affect LSPC growth [47, 48].
The stimulating factors in ECM promoting LSPC response in liver regeneration

In addition to the cross talking among cells in the LSPC niche, ECM can also be a factor that regulates LSPC activity and function [38, 49-51]. Recent studies have shown a requirement for matrix remodeling by MMPs for the proliferation of LSPCs [52]. What's more, in ECM, a range of growth factors [33, 34] including HGF, epidermal growth factor (EGF), TGF- $\alpha$ and stem cell factor (SCF) have been shown to be important in stimulating LSPCs growth, whereas TWEAK/Fibronectin (FN) 14 and stromal-derived factor 1/CXC receptor 4 (SDF-1/CXCR4) have been shown to be important for their activation and migration respectively. Multiple cytokines are involved in the activation and proliferation of LSPCs (Figure 3B). These cytokines include TNF [42], lymphotoxin alpha [53], lymphotoxin beta [54], interferon gamma (IFN- $\gamma$ ) [54], IL-22 [55], and IL-6 [56]. Two growth factors, fibroblast growth factor (FGF)7 [47] and granulocyte-colony stimulating factor (G-CSF) [57], have been proposed to act as mitogens for LSPCs. Among these cytokines and growth factors, HGF is considered to be the important. HGF/c-Met supports a pleiotrophic signal transduction pathway that controls LSPCs homeostasis [33]. Loss of c-Met receptor decreases the sphere-forming capacity of LSPCs in vitro and reduces LSPC pool, impaires migration, and decreases hepatocytic differentiation in vivo. These results establish a direct contribution of c-Met in the regulation of LSPC response and support a unique role for HGF/c-Met as an essential growth-factor-signaling pathway for regeneration of diseased liver. Another key regulatory mechanism for LSPC response is EGF/EGF receptor (EGFR). Both HGF/c-Met and EGF/EGFR collaborate to increase

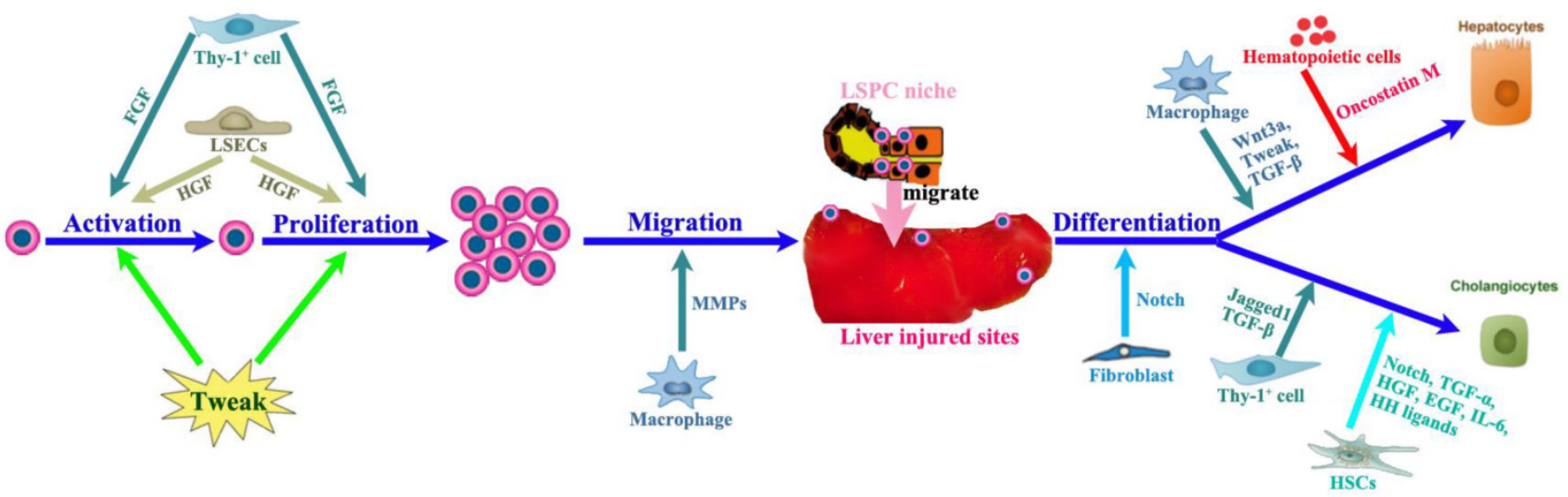

Figure 3. The impact of LSPC-niche on LSPCs mediated liver regeneration. The LSPCs mediated liver regeneration involves four consecutive processes including activation, proliferation, migration and differentiation into hepatocytes and cholangiocytes. Thy- 1 positive cells play a critical role in each step of LSPCs reaction by producing cytokines or intercellular crosstalk. Among those cells affecting LSCPs response, LSECs and SEPCs are very important, because they not only take responsibility for vasiformation, but also contribute to major HGF production. In addition, hepatic stellate cells and macrophages can send signals directly to stimulate LSPCs response to liver injury. 
the self-renewal capacity of LSPCs through activation of the extracellular signal-regulated kinase pathway (ERK) [58]. The c-Met is a strong inducer of hepatocyte differentiation by activating protein kinase $\mathrm{B}$ (PKB or AKT) and signal transducer and activator of transcription (STAT3). Conversely, EGFR selectively induced Notch1 to promote cholangiocyte specification and branching morphogenesis while concomitantly suppressing hepatocyte commitment.

The inflammatory infiltrate may also provide paracrine signals from growth and chemotactic factors to initiate the regenerative response. Potentially important elements of the inflammatory response that may stimulate LSPCs include IL-6 family [leukaemia inhibitory factor (LIF) and oncostatin M (OSM)], lymphotoxin-beta, IFN- $\gamma$ and TNF superfamily including TNF-alpha and TWEAK [19]. One of the best-established cytokines among these is TWEAK, which is produced by macrophages and has great paracrine effect on LSPCs activation and expansion [59-62].

The underlying signaling pathways during LSPC response in liver regeneration

LSPCs mediated liver regeneration is followed by a series of events involving multiple signaling pathways controlled by the previously mentioned growth factors and their receptors. The involvement of Wnt, Notch and Hedgehog ( $\mathrm{Hh})$ signalling pathways in LSPCs activation in humans has also been reported recently [63, 64]. Activation of Notch and Wnt pathways by the surrounding niche has been shown to play a critical role in the determination of LSPCs to hepatocytic and cholangiocytic fates [23, 40, 65]. There are results implying that the Wnt pathway is involved in directing LSPCs toward the hepatocytic fate, while the Notch pathway promotes differentiation of LSPCs toward the biliary lineage $[23,40,66]$. The Notch ligand Jagged1, generated by Thy1+ fibroblasts, acts on the Notch receptor expressed on LSPCs to activate the downstream signaling pathway, leading to differentiation of LPCs to cholangiocytes [40]. In contrast, the canonical Wnt molecule acts on LSPCs to induce beta-catenin signaling and expression of a Notch signal inhibitor, Numb. This eventually results in suppression of the default Notch signaling for biliary differentiation in LSPCs and, in turn, stimulates their differentiation to hepatocytes [40]. In addition, as the Wnt target gene, Lgr5 marks actively dividing stem cells in liver regeneration. In fact, Lgr5+ small cells can give birth to both hepatocytes and biliary duct cells during the repair phase [29]. Thus, the balance between two distinct niche structures with different signals is critical to shape the outcome of activated LSPCs to induce proper regenerative response according to the nature of liver injury. As a upstream functional important effector of Notch-signaling pathway, Hippo pathway signaling in vivo is sufficient to differentiate LSPCs into hepatocytes [67].

\section{The remaining questions and perspectives in LSPC mediated liver regeneration}

The pertinent activation of LSPCs is safe and necessary to help restoring severely damaged liver, however, whether LSPCs could be over-activated and impair normal liver regeneration remains unclear. Recently, it has been demonstrated that repeated LSPC reactions are not necessarily carcinogenic [20]. That is to say, the expansion of LSPC compartment conceptually can be used to facilitate liver regeneration without an increased risk of tumorigenesis.

The utilization of LSPCs for cell therapy is an attractive alternative to organ transplantation for the treatment of liver disease. There are several advantages to using LSPCs instead of primary hepatocytes or whole organ transplantation. First, a single LSPC can be expanded in culture without losing its bidirectional differentiation potential [68]. Second, the fact that LSPCs are found in the liver with various types of disorders suggests that LSPCs can be isolated from diseased livers that cannot be used for organ transplantation [69]. Third, it might be possible to isolate LSPCs from a patient who has liver disease, expand them in culture, and transplant back to the patient. This autologous transplant protocol would obviate the need for immunosuppression after transplantation. Finally, LSPCs are smaller than hepatocytes, and it has been suggested that smaller cells might have less of a tendency to cause portal hypertension following injection into the portal vein. In short, isolation and transplantation of LSPCs could represent a new approach for therapy of end-stage chronic liver diseases, as they offer many advantages to transplantation of mature hepatocytes.

\section{The contributing roles and mechanisms for SEPCs participating in liver regeneration}

\section{The contribution of LSECs in aiding liver regeneration}

Liver regeneration requires spatiotemporally precisely coordinated proliferation of the two major liver cell populations, hepatocytes and LSECs, to reconstitute liver structure and function [70]. LSECs control liver regeneration in a much more dynamic manner through stimulatory and inhibitory effects. LSECs support hepatocytes in resting liver and 
proliferate during liver regeneration to revascularize regenerated liver parenchyma [9]. In the early phases of physiological liver regeneration, inhibitor of DNA-binding protein (Id) 1 mediates inductive angiogenesis in LSECs through producing angiocrine factors Wnt2 and HGF, which provokes hepatic proliferation [71]. Notably, there is a counterbalance between activation of CXCR7-Id1 pathway in LSECs and CXCR4 upregulation after chronic injury. When CXCR7-Id1 pathway overwhelms CXCR4 pathway, it leads to normal liver regeneration. In contrast, after iterative stimuli, predominance of CXCR4 upregulation over CXCR7-Id1 activation causes fibrosis [2]. Collectively, these data shed fundamental insights into the role of the endothelium as a gatekeeper and regulator of liver regeneration [70].

\section{The contribution of sinusoidal endothelial cell progenitor cells (SEPCs) in restoring injured liver}

Recent studies indicate that not LSECs but SEPCs express more HGF and played more important roles in liver regeneration [10,72]. Two populations of SEPCs have been identified: BM SEPCs and resident or intrahepatic SEPCs (Figure 4) [9, 10,73]. Within the liver, a putative resident SEPC population has been identified based on its characteristics as a label-retaining cell. Although the logical function for the resident SEPCs would be to give rise to LSECs in normal turnover, such functionality has yet to be demonstrated. Both resident and BM SEPCs are positive for the progenitor cell marker CD133 and, like the LSECs, they express the endothelial cell marker CD31 and the hematopoietic cell marker CD45
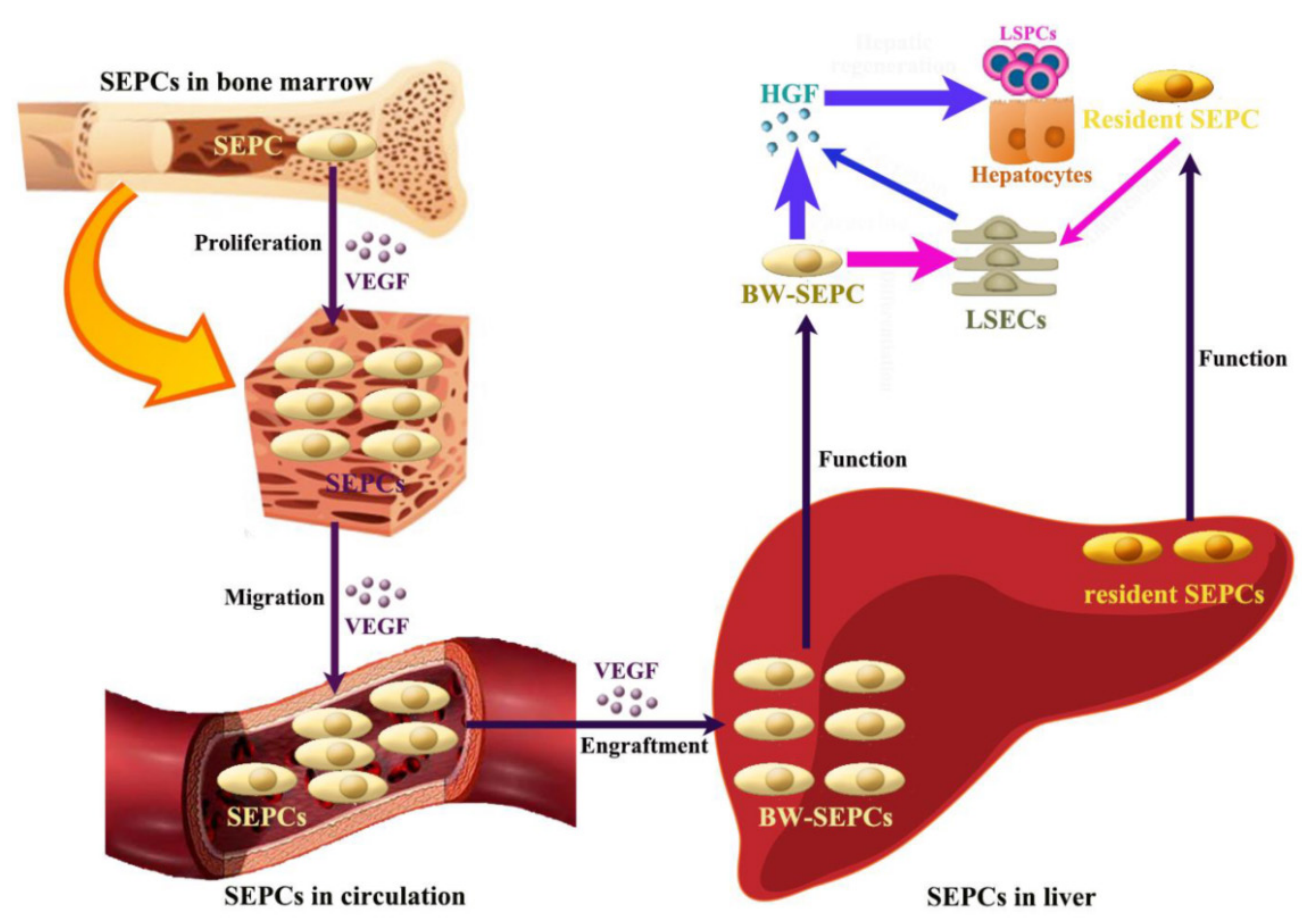

[9], also positive for vascular endothelial growth factor (VEGF) receptor (VEGFR)1 and VEGFR2. After both toxic injury and PHx, hepatic VEGF has been shown to regulate each step of BM SEPC recruitment to the liver: proliferation of BM SEPCs, mobilization of BM SEPCs to the circulation, engraftment of BM SEPCs in the liver, and differentiation of BM SEPCs to fenestrated LSECs lining the sinusoids $[9,10,73]$.

After PHx or toxic injury, proliferation of BM SEPCs increases more than two-fold and mobilization of BM SEPCs to the circulation increases two- to four-folds [9]. Thus, the BM SEPC response is a repair response to disparate forms of liver injury. It is believed that BM SEPCs that have been recruited to the liver, rather than mature LSECs, are the major drivers of liver regeneration (Figure 4) [10]. Compared to the resident SEPCs mainly contributing to normal LSEC turnover in the liver [10], BM SEPCs proliferation and mobilization to the circulation doubled and differentiated into fenestrated LSECs comprising one-quarter of the total LSECs after liver injury. What's more, BM SEPCs that engraft in the liver proliferate much more than resident SEPCs and are the major source of increased HGF in the LSEC fraction [9]. It seems that when present in their niche, resident SEPCs play a lesser role in LSEC repopulation after liver injury than BM SEPCs (Figure 4). However, when resident SEPCs are isolated from the liver and infused after $\mathrm{PHx}$, there is marked expansion of the SEPCs with persistent engraftment as LSECs and SEPCs. In addition, whether the mature LSEC also proliferates or whether all of the proliferation is attributable to resident and BM SEPCs remains to be determined. To summarize, after liver injury, BM SEPCs mainly repopulate the sinusoid as LSECs providing HGF to promote hepatocyte restoration [74].

Figure 4. The contributing roles of SEPCs during liver regeneration. There are two types of SEPCs including bone marrow derived SEPCS (BM SEPCs) and resident SEPCs existing in the liver. Under VEGF stimulation, BM SEPCs obtain increased proliferation, mobilize into circulation, and transplant in remaining liver tissue to help restoration. The recruitment of SEPCs from bone marrow are more likely to replenish LSECs for vasiformation than resident SEPCs. What's more, BM SEPCs not resident SEPCs mainly secreting cells of HGF. The HGF released by SEPCs and LSECs is the key factor for LSPCs or hepatocytes responses. 


\section{The contributing roles and mechanisms for other BM stem cells participating in liver regeneration}

The contribution to liver regeneration from different kinds of extrahepatic stem cells migrating to the injured liver such as HSCs and MSCs has been previously demonstrated [75]. BM HSCs are mobilized after liver resection, limit IL-1 $\beta$ signaling mediated inflammation, and boost regeneration in a CD39-dependent manner [76]. Due to their great contribution in liver repair, currently available HSCs mobilizing agents (G-CSF, Plerixa or G-CSF+Plerixa), were comparatively tested for healing potential in liver fibrosis [77]. In comparison to BM HSCs, BM MSCs exhibit a greater homing capability for the injured liver and modulate fibrosis and inflammation more effectively [78]. However, synergistic effects of MSCs and HSCs were not observed in liver injury.

Among immune regulating cells, both MSC and $\mathrm{T}$ regulatory (Treg) cells have been considered as possible therapeutic agents due to their ability to suppress inflammation, which is seen in many forms of chronic liver injury [79]. MSCs participate in liver regeneration in several different aspects (Figure 5). (1) MSCs help restore injured liver mass through paracrine factors: CXCR4 overexpression enhanced the mobilization and engraftment of MSCs into small-for-size liver grafts, in which these cells promoted the early regeneration of the remnant liver perhaps by a paracrine mechanism [80]. (2) MSCs release exosomes to initiate protective responses: Recently, MSC-conditioned medium (MSC-CM) containing exosomes has been shown to have protective effects against various liver injury models. MSC-derived exosomes can elicit hepatoprotective effects against injury, mainly through activation of proliferative and regenerative responses [81]. (3) MSCs could trans-differentiate into hepatocytes under certain inductive conditions: It has been hypothesized that under the influence of liver-regenerating cues, BM-MSCs can directly differentiate into hepatocytes [82]. (4) MSCs have the power to stimulate VEGF generation for vasiformation: BM-MSCs have been reported to facilitate liver regeneration after toxic injuries and massive hepatectomy, possibly by upregulating the level of VEGF [83]. (5) MSCs regulate inflammatory reaction after liver injury: MSCs promote liver repair by systemically concomitant mechanisms involving hypoxia-inducible factor-1alpha (HIF-1a) and autophagy [84].

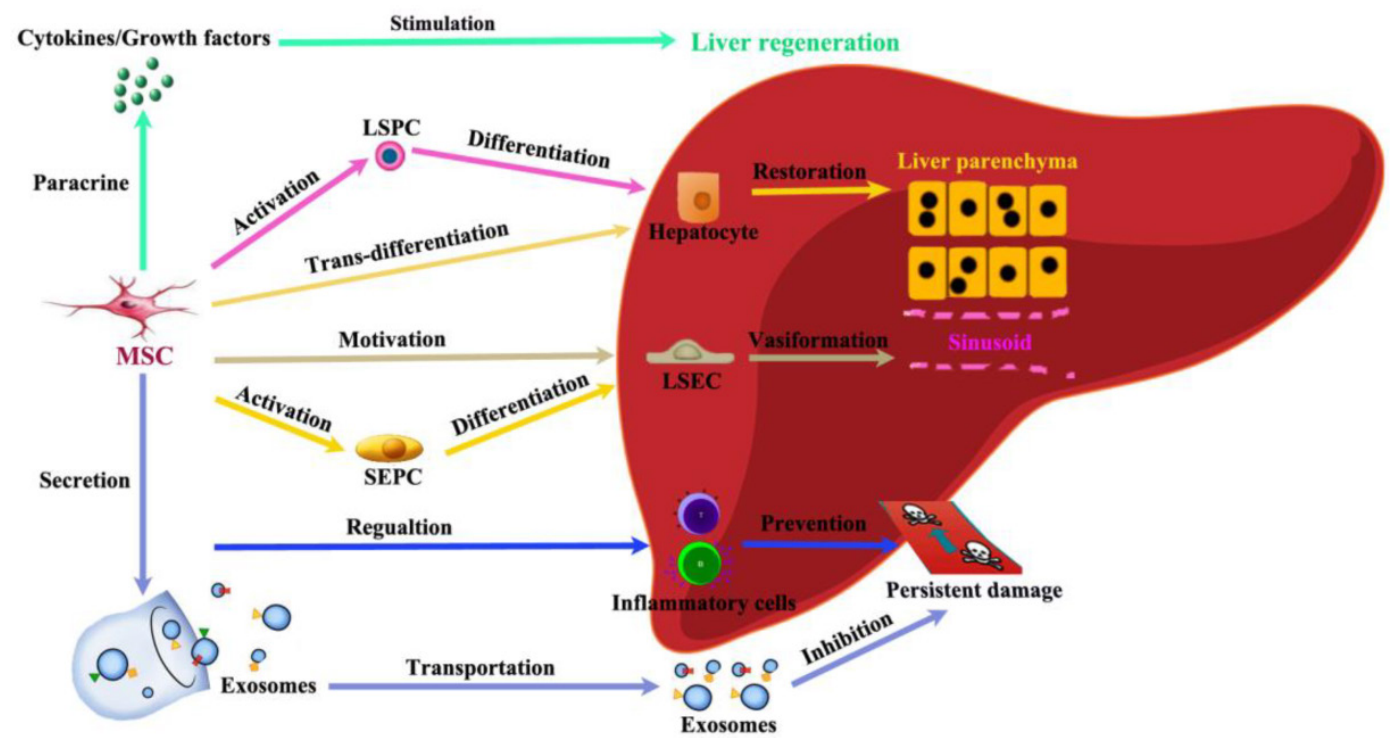

Figure 5. Schematic depicting the contributions of MSCs to liver regeneration. Liver injury induces proliferation and migration of MSCs. The recruitment of MSCs in the liver in turn actively participate in liver regeneration in several different ways: paracrine effective factors, release exosomes to initiate protective responses, trans-differentiate into hepatocytes, stimulate vasiformation, regulate inflammatory reaction.

\section{Problems and perspectives}

\section{Remaining questions}

Although the contributing roles of different types of SPCs have been greatly elucidated in liver regeneration and summarized in this review, the underlying detailed mechanism is still far from clear. There are several fundamental and critical issues concerning SPCs involved in liver regeneration that need to be addressed. First of all, the identification and isolation of SPCs remains a challenge. In spite of some potential markers being used to detect or isolate SPCs in the liver, there are some variations among 
species, especially for LSPCs. In addition, the existing markers are not specific enough for investigating subsets of SPCs, such as SEPCs. Moreover, the localization and origination of most SPCs is waiting to be thoroughly investigated. Secondly, the complete function of SPCs involved in stem cell mediated liver regeneration is pending to be explained: it is not clear if they restore damaged liver mass by differentiation into parenchymal or non-parenchymal liver cells, or by promoting regeneration and reducing damage via secreting cytokines/growth factors. Thirdly, the activating order of both intrahepatic stem cells and extrahepatic stem cells needs to be clarified, thus we can make these stem cells coordinate well and orchestrate liver regeneration. Last but not the least, it is imperative to determine the cross-talk among all these SPCs including LSPCs, SEPCs, MSCs and HSCs. When the above mentioned questions are totally answered, a proper liver regeneration could be achieved, without having the risk of over or deficient restoration that could lead to fibrosis or liver failure respectively.

\section{Potential perspectives}

Two broad strategies have been employed to enhance liver regeneration: (i) administration of stimulating factors such as G-CSF to enhance endogenous SPCs regeneration and (ii) infusions of exogenous SPCs to drive regeneration. In light of the current results, improvement of these therapies may require the co-infusion of hepatocytes/LSPCs with SECs/SEPCs. Moreover, the administration of autologous or allogeneic HSCs or/and MSCs can augment liver repair by either stimulating endogenous repair mechanisms or by suppressing ongoing damage. In addition, combining liver cells with a mimetic scaffold is also very promising [85].

As an attractive technique for scaffold preparation, decellularization can guarantee the resulting material possible to retain the liver architecture, native vessel network and specific ECM [85]. Because the SPCs-based therapies are critically dependent on the appropriate lineage differentiation of the SPCs with a high efficiency and on a considerable scale, decellularized liver bio-scaffold may provide a three-dimensional extracellular matrix for the lineage restriction of SPCs maturation. Thus, functional SPCs composite decellularized scaffolds is promising to reconstruct liver tissue. Since promotion of liver regeneration can benefit from the in vitro expansion of intrahepatic and extrahepatic SPCs, specific and safe genetic modifications aiming at these SPCs may become feasible and helpful. Once these SPCs are well modified, they can more actively involve in liver regeneration. Shortly, as the roles of cell therapies in promoting liver regeneration are gradually better understood, these therapeutic approaches will be refined and new pharmacological agents for liver repair will be developed. Nevertheless, several confronted challenges of cell therapy should be taken into consideration or discovered in the future, such as how to design a precise scheme of cell therapy based the conditions of patients, how to evaluate the effecacy of cell therapy, how to avoid possible side effects of cell therapy, and so on. In brief, our review may serve as a primary guidence for the development of regenerative strategies using SPCs during liver regeneration.

\section{Clinical importance in liver diseases}

Once we illuminate the mechanisms of SPCs mediated liver regeneration, it has very promising cilinical application in treating kinds of human liver diseases. First, for cell therapy SPCs could serve as an alternative treatment or brigde strategy for liver transplantation, especially when the donor is lack. Second, activation of endogenous SPCs is not only necessary to reserve sufficient liver function before extreme volume liver resection, but also efficient to restore damaged liver function after severe liver trama. Third, it is possible to modify liver cells under metabolic liver diseases. In detail, we can isolate SPCs from a patient who has liver disease, correct them using gene modificatin, expand them in culture, and transplant them back to the patient. Last, with the help of tissue engineering techniques, it is relatively far but very promising to rebuild a whole liver with normal function when combination of SPCs with decellularized liver scaffold. In the future, if the normally functional liver could be produced, we would have more chances to treat end-stage liver diseases.

\section{Abbreviations}

LSPCs, liver stem/progenitor cells; LSECs, liver sinusoidal endothelial cells; SEPCs, sinusoidal endothelial progenitor cells; MSCs, mesenchymal stem cells; BM, bone marrow; HSCs, hematopoietic stem cells; SPCs, stem/progenitor cells; PHx, partial hepatectomy; ECM, extracellular matrix; BM-MSCs, bone marrow MSCs; MMP, matrix metalloproteinases; HGF, hepatocyte growth factor; EGF, epidermal growth factor; TGF, transforming growth factor.

\section{Acknowledgements}

Sincere thanks go to Feng Wang (the Fourth Military Medical University, China) for renovating Figures. All authors have disclosed that they do not have any potential conflicts of interest. All authors 
have read the journal's policy on conflicts of interest. All authors have read the journal's authorship agreement.

\section{Competing Interests}

The authors have declared that no competing interest exists.

\section{References}

1. Than NN, Newsome PN. Stem cells for liver regeneration. QJM : monthly journal of the Association of Physicians. 2014;107(6):417-21.

2. Ding BS, Cao Z, Lis R, et al. Divergent angiocrine signals from vascular niche balance liver regeneration and fibrosis. Nature. 2014;505(7481):97-102.

3. Huebert RC, Shah VH. Sinusoidal endothelial cells direct traffic at the intersection of regeneration and fibrosis. Hepatology. 2014;60(2):754-6.

4. Michalopoulos GK. Advances in liver regeneration. Expert review of gastroenterology \& hepatology. 2014;8(8):897-907.

5. Kordes C, Sawitza I, Gotze S, et al. Hepatic stellate cells contribute to progenitor cells and liver regeneration. The Journal of clinical investigation. 2014;124(12):5503-15.

6. Yin C, Evason KJ, Asahina K, et al. Hepatic stellate cells in liver development, regeneration, and cancer. The Journal of clinical investigation. 2013;123(5):1902-10

7. Yin S, Wang H, Bertola A, et al. Activation of invariant natural killer $\mathrm{T}$ cells impedes liver regeneration by way of both IFN-gamma- and IL-4-dependent mechanisms. Hepatology. 2014;60(4):1356-66.

8. Yanger K, Zong Y, Maggs LR, et al. Robust cellular reprogramming occurs spontaneously during liver regeneration. Genes \& development. 2013;27(7):719-24.

9. DeLeve LD. Liver sinusoidal endothelial cells and liver regeneration. The Journal of clinical investigation. 2013;123(5):1861-6.

10. Wang L, Wang X, Xie G, et al. Liver sinusoidal endothelial cell progenitor cells promote liver regeneration in rats. The Journal of clinical investigation. 2012;122(4):1567-73.

11. Mao SA, Glorioso JM, Nyberg SL. Liver regeneration. Translational research : the journal of laboratory and clinical medicine. 2014;163(4):352-62.

12. Michalopolous G. Terminating hepatocyte proliferation during liver regeneration: the roles of two members of the same family (CCAAT-enhancer-binding protein alpha and beta) with opposing actions. Hepatology. 2015;61(1):32-4.

13. Speicher T, Siegenthaler B, Bogorad RL, et al. Knockdown and knockout of beta1-integrin in hepatocytes impairs liver regeneration through inhibition of growth factor signalling. Nature communications. 2014;5:3862

14. Itoh T, Miyajima A. Liver regeneration by stem/progenitor cells. Hepatology. 2014;59(4):1617-26.

15. Miyajima A, Tanaka M, Itoh T. Stem/progenitor cells in liver development, homeostasis, regeneration, and reprogramming. Cell stem cell. 2014;14(5):561-74.

16. Chen $\mathrm{YH}$, Chang $\mathrm{MH}$, Chien $\mathrm{CS}$, et al. Contribution of mature hepatocytes to small hepatocyte-like progenitor cells in retrorsine-exposed rats with chimeric livers. Hepatology. 2013;57(3):1215-24

17. Liu $\mathrm{WH}$, Song FQ, Ren $\mathrm{LN}$, et al. The multiple functional roles of mesenchymal stem cells in participating in treating liver diseases. Journal of cellular and molecular medicine. 2015;19(3):511-20.

18. Tanimizu N, Mitaka T. Re-evaluation of liver stem/progenitor cells. Organogenesis. 2014;10(2):208-15.

19. Katoonizadeh A, Poustchi H, Malekzadeh R. Hepatic progenitor cells in liver regeneration: current advances and clinical perspectives. Liver international : official journal of the International Association for the Study of the Liver. 2014:34(10):1464-72.

20. Papp V, Rokusz A, Dezso K, et al. Expansion of hepatic stem cell compartment boosts liver regeneration. Stem cells and development. 2014;23(1):56-65.

21. Wang B, Zhao L, Fish M, et al. Self-renewing diploid Axin2(+) cells fuel homeostatic renewal of the liver. Nature. 2015;524(7564):180-5.

22. Shin S, Kaestner KH. The origin, biology, and therapeutic potential of facultative adult hepatic progenitor cells. Current topics in developmental biology. 2014;107:269-92.

23. Liu WH, Ren LN, Chen T, et al. Unbalanced distribution of materials: the art of giving rise to hepatocytes from liver stem/progenitor cells. J Cell Mol Med. 2014;18(1):1-14.

24. Minguet S, Cortegano I, Gonzalo P, et al. A population of c-Kit(low)(CD45/TER119)- hepatic cell progenitors of 11-day postcoitus mouse embryo liver reconstitutes cell-depleted liver organoids. The Journal of clinical investigation. 2003;112(8):1152-63.

25. Suzuki A, Zheng YW, Fukao K, et al. Liver repopulation by c-Met-positive stem/progenitor cells isolated from the developing rat liver. Hepato-gastroenterology. 2004;51(56):423-6.

26. Kakinuma S, Ohta H, Kamiya A, et al. Analyses of cell surface molecules on hepatic stem/progenitor cells in mouse fetal liver. Journal of hepatology. 2009;51(1):127-38.
27. Kamiya A, Kakinuma S, Yamazaki $Y$, et al. Enrichment and clonal culture of progenitor cells during mouse postnatal liver development in mice. Gastroenterology. 2009;137(3):1114-26, 26 e1-14.

28. Qiu Q, Hernandez JC, Dean AM, et al. CD24-positive cells from normal adult mouse liver are hepatocyte progenitor cells. Stem cells and development. 2011;20(12):2177-88.

29. Huch M, Dorrell C, Boj SF, et al. In vitro expansion of single Lgr5+ liver stem cells induced by Wnt-driven regeneration. Nature. 2013;494(7436):247-50.

30. Katoonizadeh A, Nevens F, Verslype C, et al. Liver regeneration in acute severe liver impairment: a clinicopathological correlation study. Liver international : official journal of the International Association for the Study of the Liver. 2006;26(10):1225-33.

31. Oh SH, Witek RP, Bae $\mathrm{SH}$, et al. Bone marrow-derived hepatic oval cells differentiate into hepatocytes in 2-acetylaminofluorene/partial hepatectomy-induced liver regeneration. Gastroenterology. 2007;132(3):1077-87.

32. Dusabineza AC, Van Hul NK, Abarca-Quinones J, et al. Participation of liver progenitor cells in liver regeneration: lack of evidence in the $\mathrm{AAF} / \mathrm{PH}$ rat model. Laboratory investigation; a journal of technical methods and pathology. 2012;92(1):72-81.

33. Ishikawa T, Factor VM, Marquardt JU, et al. Hepatocyte growth factor/c-met signaling is required for stem-cell-mediated liver regeneration in mice. Hepatology. 2012;55(4):1215-26.

34. Jelnes P, Santoni-Rugiu E, Rasmussen M, et al. Remarkable heterogeneity displayed by oval cells in rat and mouse models of stem cell-mediated liver regeneration. Hepatology. 2007;45(6):1462-70.

35. Tonkin JN, Knight B, Curtis D, et al. Bone marrow cells play only a very minor role in chronic liver regeneration induced by a choline-deficient, ethionine-supplemented diet. Stem cell research. 2008;1(3):195-204.

36. Rehman K, Iqbal MJ, Zahra N, et al. Liver stem cells: from preface to advancements. Current stem cell research \& therapy. 2014;9(1):10-21.

37. Lanzoni G, Oikawa $T$, Wang $Y$, et al. Concise review: clinical programs of stem cell therapies for liver and pancreas. Stem cells. 2013;31(10):2047-60.

38. Lozoya OA, Wauthier E, Turner RA, et al. Regulation of hepatic stem/progenitor phenotype by microenvironment stiffness in hydrogel models of the human liver stem cell niche. Biomaterials. 2011;32(30):7389-402.

39. Gaudio E, Carpino G, Cardinale V, et al. New insights into liver stem cells. Digestive and liver disease : official journal of the Italian Society of Gastroenterology and the Italian Association for the Study of the Liver. 2009;41(7):455-62.

40. Boulter L, Govaere O, Bird TG, et al. Macrophage-derived Wnt opposes Notch signaling to specify hepatic progenitor cell fate in chronic liver disease. Nature medicine. 2012;18(4):572-9.

41. Lorenzini S, Bird TG, Boulter L, et al. Characterisation of a stereotypical cellular and extracellular adult liver progenitor cell niche in rodents and diseased human liver. Gut. 2010;59(5):645-54.

42. Viebahn CS, Benseler V, Holz LE, et al. Invading macrophages play a major role in the liver progenitor cell response to chronic liver injury. Journal of hepatology. 2010;53(3):500-7.

43. Sackett SD, Li Z, Hurtt R, et al. Foxl1 is a marker of bipotential hepatic progenitor cells in mice. Hepatology. 2009;49(3):920-9.

44. Schotanus BA, van den Ingh TS, Penning LC, et al. Cross-species immunohistochemical investigation of the activation of the liver progenitor cell niche in different types of liver disease. Liver international : official journal of the International Association for the Study of the Liver. 2009;29(8):1241-52.

45. Yovchev MI, Zhang J, Neufeld DS, et al. Thymus cell antigen-1-expressing cells in the oval cell compartment. Hepatology. 2009;50(2):601-11.

46. Chen L, Zhang W, Zhou QD, et al. HSCs play a distinct role in different phases of oval cell-mediated liver regeneration. Cell biochemistry and function. 2012;30(7):588-96.

47. Takase HM, Itoh T, Ino S, et al. FGF7 is a functional niche signal required for timulation of adult liver progenitor cells that support liver regeneration. Genes \& development. 2013;27(2):169-81

48. Yovchev MI, Grozdanov PN, Zhou H, et al. Identification of adult hepatic progenitor cells capable of repopulating injured rat liver. Hepatology. 2008;47(2):636-47.

49. Carpentier R, Suner RE, van Hul N, et al. Embryonic ductal plate cells give rise to cholangiocytes, periportal hepatocytes, and adult liver progenitor cells. Gastroenterology. 2011:141(4):1432-8, 8 e1-4.

50. Van Hul N, Lanthier N, Espanol Suner R, et al. Kupffer cells influence parenchymal invasion and phenotypic orientation, but not the proliferation, of liver progenitor cells in a murine model of liver injury. The American journal of pathology. 2011;179(4):1839-50.

51. Van Hul NK, Abarca-Quinones J, Sempoux C, et al. Relation between liver progenitor cell expansion and extracellular matrix deposition in a CDE-induced murine model of chronic liver injury. Hepatology. 2009;49(5):1625-35.

52. Williams MJ, Clouston AD, Forbes SJ. Links between hepatic fibrosis, ductular reaction, and progenitor cell expansion. Gastroenterology. 2014·146(2):349-56.

53. Knight B, Yeoh GC. TNF/LTalpha double knockout mice display abnormal inflammatory and regenerative responses to acute and chronic liver injury. Cell and tissue research. 2005;319(1):61-70.

54. Akhurst B, Matthews V, Husk K, et al. Differential lymphotoxin-beta and interferon gamma signaling during mouse liver regeneration induced by chronic and acute injury. Hepatology. 2005;41(2):327-35. 
55. Feng D, Kong X, Weng $\mathrm{H}$, et al. Interleukin-22 promotes proliferation of liver stem/progenitor cells in mice and patients with chronic hepatitis B virus infection. Gastroenterology. 2012;143(1):188-98 e7.

56. Yeoh GC, Ernst M, Rose-John S, et al. Opposing roles of gp130-mediated STAT-3 and ERK-1/ 2 signaling in liver progenitor cell migration and proliferation. Hepatology. 2007;45(2):486-94.

57. Piscaglia AC, Shupe TD, Oh SH, et al. Granulocyte-colony stimulating factor promotes liver repair and induces oval cell migration and proliferation in rats. Gastroenterology. 2007;133(2):619-31.

58. Kitade M, Factor VM, Andersen JB, et al. Specific fate decisions in adult hepatic progenitor cells driven by MET and EGFR signaling. Genes \& development. 2013;27(15):1706-17.

59. Tirnitz-Parker JE, Viebahn CS, Jakubowski A, et al. Tumor necrosis factor-like weak inducer of apoptosis is a mitogen for liver progenitor cells. Hepatology. 2010;52(1):291-302.

60. Jakubowski A, Ambrose C, Parr M, et al. TWEAK induces liver progenitor cell proliferation. The Journal of clinical investigation. 2005;115(9):2330-40.

61. Chazaud B. Macrophages: supportive cells for tissue repair and regeneration. Immunobiology. 2014;219(3):172-8.

62. Bird TG, Lu WY, Boulter L, et al. Bone marrow injection stimulates hepatic ductular reactions in the absence of injury via macrophage-mediated TWEAK signaling. Proceedings of the National Academy of Sciences of the United States of America. 2013;110(16):6542-7.

63. Roskams T, Katoonizadeh A, Komuta M. Hepatic progenitor cells: an update. Clinics in liver disease. 2010;14(4):705-18.

64. Spee B, Carpino G, Schotanus BA, et al. Characterisation of the liver progenitor cell niche in liver diseases: potential involvement of Wnt and Notch signalling. Gut. 2010;59(2):247-57.

65. Wang T, Chen T, Liang HY, et al. Notch inhibition promotes fetal liver stem/progenitor cells differentiation into hepatocytes via the inhibition of HNF-1beta. Cell and tissue research. 2014;357(1):173-84

66. Strazzabosco M, Fabris L. The balance between Notch/Wnt signaling regulates progenitor cells' commitment during liver repair: mystery solved? Journal of hepatology. 2013;58(1):181-3.

67. Yimlamai D, Christodoulou C, Galli GG, et al. Hippo pathway activity influences liver cell fate. Cell. 2014;157(6):1324-38.

68. Shin S, Walton G, Aoki R, et al. Foxl1-Cre-marked adult hepatic progenitors have clonogenic and bilineage differentiation potential. Genes \& development. 2011;25(11):1185-92.

69. Nobili V, Carpino G, Alisi A, et al. Hepatic progenitor cells activation, fibrosis, and adipokines production in pediatric nonalcoholic fatty liver disease. Hepatology. 2012;56(6):2142-53.

70. Hu J, Srivastava K, Wieland M, et al. Endothelial cell-derived angiopoietin-2 controls liver regeneration as a spatiotemporal rheostat. Science. 2014;343(6169):416-9.

71. Moniaux N, Faivre J. Key role of sinusoidal endothelial cells in the triggering of liver regeneration. Journal of hepatology. 2011;55(2):488-90.

72. Wang R, Huebert RC, Shah VH. Sinusoidal endothelial cells coordinate liver regeneration and angiogenesis via angiopoietin-2: an ode to prometheus. Gastroenterology. 2014;147(2):533-4.

73. DeLeve LD. Liver sinusoidal endothelial cells in hepatic fibrosis. Hepatology. 2015;61(5):1740-6.

74. Wang L, Wang X, Wang L, et al. Hepatic vascular endothelial growth factor regulates recruitment of rat liver sinusoidal endothelial cell progenitor cells. Gastroenterology. 2012;143(6):1555-63 e2.

75. Okabayashi T, Shima Y, Sumiyoshi T, et al. Extrahepatic stem cells mobilized from the bone marrow by the supplementation of branched-chain amino acids ameliorate liver regeneration in an animal model. Journal of gastroenterology and hepatology. 2014;29(4):870-7.

76. Schmelzle M, Duhme C, Junger W, et al. CD39 modulates hematopoietic stem cell recruitment and promotes liver regeneration in mice and humans after partial hepatectomy. Annals of surgery. 2013;257(4):693-701.

77. Tsolaki E, Athanasiou E, Gounari E, et al. Hematopoietic stem cells and liver regeneration: differentially acting hematopoietic stem cell mobilization agents reverse induced chronic liver injury. Blood cells, molecules \& diseases. 2014;53(3):124-32.

78. Li Q, Zhou X, Shi $Y$, et al. In vivo tracking and comparison of the therapeutic effects of MSCs and HSCs for liver injury. PloS one. 2013;8(4):e62363.

79. Zhang Y, Cai W, Huang $Q$, et al. Mesenchymal stem cells alleviate bacteria-induced liver injury in mice by inducing regulatory dendritic cells. Hepatology. 2014;59(2):671-82.

80. Fouraschen $\mathrm{SM}$, Pan $\mathrm{Q}$ de Ruiter $\mathrm{PE}$ et al Secreted factors of human liver-derived mesenchymal stem cells promote liver regeneration early after partial hepatectomy. Stem cells and development. 2012;21(13):2410-9.

81. Tan CY, Lai RC, Wong W, et al. Mesenchymal stem cell-derived exosomes promote hepatic regeneration in drug-induced liver injury models. Stem cell research \& therapy. 2014;5(3):76.

82. Mukhopadhyay A. Perspective on liver regeneration by bone marrow-derived stem cells-a scientific realization or a paradox. Cytotherapy. 2013;15(8):881-92.

83. Yu J, Yin $\mathrm{S}$, Zhang $\mathrm{W}$, et al. Hypoxia preconditioned bone marrow mesenchymal stem cells promote liver regeneration in a rat massive hepatectomy model. Stem cell research \& therapy. 2013;4(4):83.

84. Jung J, Choi JH, Lee Y, et al. Human placenta-derived mesenchymal stem cells promote hepatic regeneration in CCl4 -injured rat liver model via increased autophagic mechanism. Stem cells. 2013;31(8):1584-96.
85. Zhou Q Li L, Li J. Stem cells with decellularized liver scaffolds in liver regeneration and their potential clinical applications. Liver international : official journal of the International Association for the Study of the Liver. 2015;35(3):687-94. 